\title{
ONE-SIDED IDEALS AND DERIVATIONS OF PRIME RINGS
}

\author{
MATEJ BREŠAR
}

(Communicated by Maurice Auslander)

\begin{abstract}
The action of derivations on right ideals of prime rings is considered.
\end{abstract}

\section{INTRODUCTION AND STATEMENT OF THE RESULTS}

Over the last twenty years there has been a great deal of work concerning derivations of prime rings. A number of authors have proved theorems which, roughly speaking, show that the existence of a derivation satisfying certain relations implies that either the ring or the derivation is very special. Also, there are many results giving conditions under which a derivation of a prime ring can be completely determined by the action on some subsets of the ring (such as (two-sided) ideals, Lie ideals, symmetric or skew elements of a ring with involution, etc.). The purpose of this note is to look at the action of derivations on one-sided ideals.

The following three theorems present a motivation for the results in this paper.

Let $R$ be a prime ring, $I$ be a nonzero (two-sided) ideal of $R$, and $d$ be a derivation of $R$. Then:

(A) If $d^{3} \neq 0$, then the subring generated by $d(I)$ contains a nonzero ideal of $R$.

(B) If char $R \neq 2$ and $\delta$ is another derivation of $R$ such that $\delta d(I)=0$, then either $d=0$ or $\delta=0$.

(C) If there exists a positive integer $n$ such that $d(x)^{n}=0$ for all $x \in I$, then $d=0$.

The fundamental case of (A) when $I=R$ was proved by Herstein [7, Theorem 1]; a generalization to Lie ideals was obtained by Bergen, Herstein, and Kerr [1, Theorem 3]. The study of compositions of derivations was initiated by a well-known theorem of Posner [10, Theorem 1] which is related to (B). A generalization of (B) to Lie ideals can be, for instance, found in [1, Theorem 4]. (C) is due to Giambruno and Herstein [4, Theorem 2] (see also [2, 3]).

Received by the editors March 13, 1992 and, in revised form, March 10, 1993. 1991 Mathematics Subject Classification. Primary 16W25; Secondary 16N60.

Key words and phrases. Derivation, prime ring, right ideal.

Supported by the Research Council of Slovenia. 
By simple examples one can show that under the assumption that $I$ is merely a right ideal, none of the conclusions of the above results holds. It turns out that in this case the adequate conclusions are closely connected with the condition $d(I) I=0$. This condition is the main object of this paper.

Notation. Henceforth $R$ will be a prime ring with a derivation $d$. By $U$ we denote a right ideal of $R$, and by $L$ the left annihilator of $U$ (i.e., $L=\{x \in$ $R \mid x U=0\}$ ). The (right) Martindale ring of quotients of $R$ will be denoted by $Q$.

For the notion of the Martindale ring of quotients see [6,9].

We remark that the condition $d(U) U=0$ can be written in the form $d(U) \subseteq$ $L$. Our first result is a simple observation showing that this condition can be characterized in four ways.

Lemma. Suppose $U \neq 0$. Then the following conditions are equivalent:

(i) $d(U) \subseteq L$;

(ii) $d(U)^{2}=0$;

(ii) $d(U) a=0$ for some nonzero $a \in R$;

(iv) $d=[q, \cdot]$ for some $q \in Q$ such that $q U=0$.

By $d=[q, \cdot]$ we mean that $d(x)=[q, x]=q x-x q$ for all $x \in R$ (that is, $d$ is Kharchenko inner).

Proof of Lemma. Suppose condition (i) holds; that is, $d(U) U=0$. Whence $0=d(U d(U)) U=U d^{2}(U) U$. Since $R$ is prime, it follows that $d^{2}(U) U=0$. Consequently, $d(U)^{2}=0$ results from $d(d(U) U)=0$. Thus (i) implies (ii). Clearly, a nonzero derivation cannot vanish on a nonzero right ideal of a prime ring. Therefore, (ii) implies (iii). It is very easy to show that (iii) implies (i). For the proof of the equivalence of (iii) and (iv) see [8] where an analogous result for left ideals is proved.

The goal of this paper is to prove the following three theorems which extend (A), (B), and (C).

Theorem 1. Suppose char $R \neq 2$. Then the subring of $R$ generated by $d(U)$ contains no nonzero right ideals of $R$ if and only if $d(U) \subseteq L$.

Theorem 2. Suppose $U \neq 0, d \neq 0$, char $R \neq 2$, and $\delta \neq 0$ is another derivation of $R$. Then $\delta d(U)=0$ if and only if $d(U) \subseteq L, \delta(U) \subseteq L$, and there exist $q, p \in Q$ such that $d=[q, \cdot], \delta=[p, \cdot], q U=0, p U=0$, and $q p=0$.

Theorem 3. Suppose char $R=0$. Then there exists an integer $n>1$ such that $d(u)^{n} \in L$ for all $u \in U$ if and only if $d(U) \subseteq L$.

\section{Proofs}

Proof of Theorem 1. Let $S$ be the subring generated by $d(U)$. First suppose $d(U) U=0$. Then $S U=0$, and so, by the primeness of $R, S$ cannot contain nonzero right ideals.

To prove the reverse implication, we set $T=S \cap U$. For $t \in T, u \in U$, we have $d(t) u=d(t u)-t d(u) \in S$. Thus, $d(T) U$ is a right ideal of $R$ contained 
in $S$. Therefore, the theorem will be proved by showing that $d(T) U=0$ (i.e., $d(T) \subseteq L)$ implies $d(U) \subseteq L$.

Pick $u \in U, s \in S$. As $u d(s)=d(u s)-d(u) s$, we have $u d(s) \in T$. Applying $d(T) \subseteq L$ we then get $d(u) d(s)+u d^{2}(s) \in L, u \in U, s \in S$. In view of the identity

$$
d(u) v d(s)=d(u v) d(s)+(u v) d^{2}(s)-u\left\{d(v) d(s)+v d^{2}(s)\right\}
$$

we see that $d(u) v d(s) \in L, u, v \in U, s \in S$. That is, $d(U) U d(S) U=0$. Since $R$ is prime, either $d(U) U=0$ or $d(S) U=0$. Of course, we may assume that $d(S) U=0$, i.e., $d(S) \subseteq L$. In particular, $d^{2}(u) \in L$ for every $u \in U$. Hence $2 d(u) d(v)=d^{2}(u v)-d^{2}(u) v-u d^{2}(v) \in L, u, v \in U$, and therefore, since char $R \neq 2, d(u) d(v) \in L$. Consequently, $d(u) w d(v)=$ $d(u w) d(v)-u d(w) d(v) \in L, u, w, v \in L$. Thus $d(U) U d(U) U=0$ which yields $d(U) U=0$. The proof of the theorem is complete.

Remark 1. The assumption in Theorem 2 that char $R \neq 2$ cannot be removed. Namely, consider the following example. Suppose a prime ring $R$ with char $R=$ 2 contains an element $a$ such that $a^{\ddot{\alpha}}=0$ and $a$ does not lie in the center of $R$ (cf. [6, p. 10]). Let $d=[\cdot, a]$. Then $d \neq 0$ so that $d(R) R \neq 0$. Since $d^{2}(R)=0$ it follows that $d(S)=0$, where $S$ is a subring generated by $d(R)$. But then $S$ cannot contain nonzero right ideals.

Proof of Theorem 2. Assume that $\delta d(U)=0$. Then $\delta(S)=0$, where $S$ is a subring generated by $d(U)$. Since $\delta \neq 0, S$ cannot contain nonzero right ideals of $R$. By Theorem 1 it follows that $d(U) \subseteq L$. Whence $d\left(U^{2}\right)=$ $U d(U)$, and therefore, $0=\delta d\left(U^{2}\right)=\delta(U d(U))=\delta(U) d(U)$. As $d \neq 0$ and $U \neq 0$ we have $d(U) \neq 0$, so the Lemma tells us that $\delta(U) \subseteq L$.

By the Lemma there exist $p, q \in Q$ such that $d=[q, \cdot], \delta=[p, \cdot]$, $q U=0$, and $p U=0$. Hence, for $u \in U$ we have $0=\delta d(u)=u p q$. Since $R$ is prime it follows that $q p=0$.

The reverse implication is trivial.

Remark 2. In Theorem 1 we showed that there are derivations $d$ of prime rings $R$ with $\operatorname{char} R=2$ such that $d^{2}(R)=0$ but $d(R) R \neq 0$. This justifies the necessity of the assumption char $R \neq 2$ in Theorem 2 .

We also remark that Theorem 2 could be proved more directly (without using Theorem 1) by adapting the arguments of Posner [10].

Proof of Theorem 3. Our goal is to show that $d(u)^{n} \in L, u \in U$, implies $d(u)^{n-1} \in L, u \in U$.

First we prove that $L d(U)=0$. By assumption, for every $a \in R$ we have $d(u a)^{n} u=0, u \in U$. If $a \in L$ then $d(u a) u=d(u) a u+u d(a) u=u d(a) u$, and therefore $(u d(a))^{n} u=0, u \in U$. Hence $(d(a) u)^{n+1}=0, u \in U$. That is, every element $w$ from the right ideal $d(a) U$ satisfies $w^{n+1}=0$. By a result of Levitzki [5, Lemma 1.1] it follows that $d(a) U=0$. Since $a u=0, u \in U$, it follows that $a d(u)=d(a u)-d(a) u=0$, as claimed.

A simple modification of the proof of [3, Lemma 2] shows that

$$
\sum_{k=0}^{n-1} d(u)^{k} d(v) d(u)^{n-k-1} \in L
$$


for all $u, v \in U$. Replacing $v$ by $v d(u)$ it follows that

$$
\left\{\sum_{k=0}^{n-1} d(u)^{k} d(v) d(u)^{n-k-1}\right\} d(u)+\sum_{k=0}^{n-1} d(u)^{k} v d^{2}(u) d(u)^{n-k-1} \in L .
$$

Since $L d(U)=0$, the first summand equals zero. Hence

$$
\sum_{k=0}^{n-1} d(u)^{k} v d^{2}(u) d(u)^{n-k-1} \in L
$$

for all $u, v \in U$. As $L$ is a left ideal, we also have

$$
d(u)^{n-1}\left(\sum_{k=0}^{n-1} d(u)^{k} v d^{2}(u)(u)^{n-k-1}\right) \in L
$$

since $d(u)^{n} U=0$, it follows that $d(u)^{n-1} v d^{2}(u) d(u)^{n-1} \in L$. Suppose that $d(u)^{n-1} U \neq 0$ for some $u \in U$; by the primeness of $R$ we then get $d^{2}(u) d(u)^{n-1}$ $\in L$. Therefore, (1) reduces to

$$
\sum_{k=0}^{n-1} d(u)^{k} v d^{2}(u) d(u)^{n-k-1} \in L,
$$

$v \in U$. Multiplying this element from the left by $d(u)^{n-2}$ we arrive at

$$
d(u)^{n-1} v d^{2}(u) d(u)^{n-2} \in L, \quad v \in U,
$$

which yields $d^{2}(u) d(u)^{n-2} \in L$. Thus, the first summand in (2) is contained in $L$. We continue this procedure and finally reach $d(u)^{n-1} v d^{2}(u) \in L$ for every $v \in U$. Whence $d^{2}(u) \in L$.

Thus, we have proved that for any $u \in U$ either $d(u)^{n-1} \in L$ or $d^{2}(u) \in L$. Suppose there exist $u, v \in U$ such that $d(u)^{n-1} \notin L$ and $d^{2}(v) \notin L$. Hence $d^{2}(u) \in L$, and therefore, $d^{2}(u+k v) \notin L$ for every integer $k$. Consequently, $d(u+k v)^{n-1} \in L$ for every $k$, i.e., $(d(u)+k d(v))^{n-1} U=0$. As $d(v)^{n-1} U=0$, this can be written in the form

$$
y_{1} w+k y_{2} w+k^{2} y_{3} w+\cdots+k^{n-2} y_{n-1} w=0
$$

for every integer $k$, where $w$ is an arbitrary element in $U$ and

$$
\begin{gathered}
y_{1}=d(u)^{n-1}, \\
y_{2}=\sum_{k=0}^{n-2} d(u)^{k} d(v) d(u)^{n-k-2}, \\
\vdots \\
y_{n-1}=\sum_{k=0}^{n-2} d(v)^{k} d(u) d(v)^{n-k-2} .
\end{gathered}
$$

By [3, Lemma 1] we have $y_{i} w=0, i=1,2, \ldots, n-1$. In particular, $d(u)^{n-1} w=0, w \in U$, contrary to the assumption. This proves that either $d(u)^{n-1} \in L$ for every $u \in U$ or $d^{2}(u) \in L$ for every $u \in U$. However, if the latter occurs, then we have $2 d(u) d(v)=d^{2}(u v)-d^{2}(u) v-u d^{2}(v) \in L$, 
$u, v \in U$; hence $d(u) d(v) \in L$, and therefore, $d(u) w d(v)=d(u w) d(v)-$ $u d(w) d(v) \in L, u, w, v \in L$. That is, $d(U) U d(U) U=0$. Since $R$ is prime it follows that $d(U) \subseteq L$. Thus, in any case we have $d(u)^{n-1} \in L, u \in U$. This proves the theorem.

Remark 3. An examination of the proof of Theorem 3 shows that (at least when $n \neq 2$ ) the assumption char $R=0$ could be replaced by a milder assumption that $R$ is $(n-1)$ !-torsion free (cf. [3]). However, probably even this assumption is redundant, but we were unable to prove this.

\section{ACKNOWLEDGEMENT}

The author would like to thank the referee for valuable suggestions.

\section{REFERENCES}

1. J. Bergen, I. N. Herstein, and J. W. Kerr, Lie ideals and derivations of prime rings, J. Algebra 71 (1981), 259-267.

2. M. Brešar, A note on derivations, Math. J. Okayama Univ. 32 (1990), 83-88.

3. L. O. Chung and J. Luh, Semiprime rings with nilpotent derivatives, Canad. Math. Bull. 24 (1981), 415-421.

4. A. Giambruno and I. N. Herstein, Derivations with nilpotent values, Rend. Circ. Mat. Palermo 30 (1981), 199-206.

5. I. N. Herstein, Topics in ring theory, Univ. of Chicago Press, Chicago, 1969.

6. __ Rings with involution, Univ. of Chicago Press, Chicago, 1976.

7. __ A note on derivations, Canad. Math. Bull. 21 (1978), 369-370.

8. _ A condition that a derivation be inner, Rend. Circ. Mat. Palermo 37 (1988), 5-7.

9. W. S. Martindale, Prime rings satisfying a generalized polynomial identity, J. Algebra 12 (1969), 576-584.

10. E. C. Posner, Derivations in prime rings, Proc. Amer. Math. Soc. 8 (1957), 1093-1100.

Department of MAThematics, University of Maribor, PF, KorošKa 160, 62000 Maribor, SLOVENIA 